\title{
Localization Effects in GaN/AlGaN Quantum Well - Photoluminescence Studies
}

\author{
B. Chwalisz ${ }^{a, *}$, A. WysmoŁeK ${ }^{a}$, R. BożeK ${ }^{a}$, K.P. Korona ${ }^{a}$, \\ R. Stępniewski ${ }^{a}, W . \mathrm{KnaP}^{b}, \mathrm{~K}$. PakuŁ $\mathrm{A}^{a}$, \\ J.M. Baranowski ${ }^{a}$, N. Grandjean ${ }^{c}$, J. Massies ${ }^{c}$, \\ P. Prystawko ${ }^{d}$ AND I. Grzegory ${ }^{d}$ \\ ${ }^{a}$ Institute of Experimental Physics, Warsaw University \\ Hoża 69, 00-681 Warsaw, Poland
}

${ }^{b}$ Groupe d'Etude des Semiconducteurs, CNRS-UMR5650 Université Montpellier2 Montpellier, France

${ }^{c}$ CNRS - CRHEA, rue Bernard Grégory, Valbonne-Sophia-Antipolis, France

${ }^{d}$ High Pressure Research Center, Polish Academy of Sciences Sokołowska 29/37, Warsaw, Poland

Exciton localization in GaN/AlGaN quantum well structures is studied by photoluminescence. An anomalous temperature behavior of the photoluminescence from the quantum well is observed. With increasing temperature the energy position of the excitonic emission line first decreases up to $20 \mathrm{~K}$, then increases, reaching a maximum around $90 \mathrm{~K}$, and then decreases again in the higher temperature range. The observed behavior is discussed in terms of localization at the interface potential fluctuations. It is argued that the temperature activated migration and subsequent release of the excitons from traps that occurs between $20 \mathrm{~K}$ and $90 \mathrm{~K}$ are responsible for the observed S-like shape of the energy dependence. The obtained results allow a direct characterization of the energy fluctuations present in GaN/AlGaN quantum wells grown by different techniques.

PACS numbers: 78.55.Cr, 73.20.-r, 73.21.-b, 74.40.+k

\section{Introduction}

The III-nitride systems are now intensively studied due to their possible applications in optical and electronic devices such as UV LEDs and HEMT tran-

*corresponding author; e-mail: Barbara.Chwalisz@fuw.edu.pl 
sistors. In particular, due to its large energy gap and high thermal stability, the GaN/AlGaN system is a promising candidate for the fabrication of high-power devices. The optical quality of GaN/AlGaN quantum wells (QWs), as determined from the emission efficiency, is still not as high as that of GaN/InGaN structures. The photoluminescence (PL) of two-dimensional heterosystems can provide important information about the mechanisms involved in the recombination processes as well as indications about the energy distribution of carriers. Temperature modifies contributions to the emission from free and localized excitons. One can expect that at low temperatures the excitons occupy lower, localized, energy states in the potential fluctuations, which contribute significantly to the luminescence. At higher temperatures emission due to the recombination of free excitons dominates the spectra. Thus, the study of the temperature dependent luminescence spectra can give quantitative information about exciton localization energy and character of potential fluctuations.

Optical investigations of localization mechanisms performed on $\mathrm{ZnSe} / \mathrm{ZnS}$ [1, 2], InGa/GaN [3] and InGaN/AlGaN [4] low-dimensional structures showed similar temperature dependent behavior. In these materials interface roughness and structural disorder plays a major role leading to the formation of objects such as quantum slabs and/or quantum dots localizing the excitons.

In this paper the influence of localization on the emission properties of $\mathrm{GaN} / \mathrm{AlGaN} \mathrm{QW}$ is presented.

\section{Experimental results and discussion}

The results of two samples containing GaN/AlGaN QW are presented. Sample A was grown by low pressure metal-organic chemical vapor deposition (MOCVD). The sapphire substrate was covered by a $40 \mathrm{~nm} \mathrm{GaN} \mathrm{buffer} \mathrm{and} \mathrm{a}$ $3 \mu \mathrm{m} \mathrm{GaN} \mathrm{layer.} \mathrm{Further} \mathrm{on,} \mathrm{the} \mathrm{structure} \mathrm{consists} \mathrm{of} \mathrm{a} \mathrm{single} 40 \AA \mathrm{QW}$ embedded between $200 \mathrm{~nm}$ and $100 \mathrm{~nm} \mathrm{AlGaN}$ barriers with an aluminum content of approximately $9 \%$. Sample B was grown by molecular beam epitaxy (MBE) on a bulk GaN substrate. First, an $0.5 \mu \mathrm{m} \mathrm{GaN} \mathrm{layer,} \mathrm{intentionally} n$-type doped (approximately $5 \times 10^{17} \mathrm{~cm}^{-3}$ ) was deposited. Further on, the structure consists of a single $20 \AA \mathrm{QW}$ embedded between $500 \AA$ AlGaN barriers with an aluminum content of approximately $9 \%$.

PL measurements were performed at temperatures from $4.2 \mathrm{~K}$ up to $220 \mathrm{~K}$ in a continuous flow cryostat. The samples were excited by the $\lambda=325 \mathrm{~nm}$ line of a $\mathrm{He}-\mathrm{Cd}$ laser (with a power of approximately $2 \mathrm{~mW}$ ). The PL signal was dispersed by a SPEX $500 \mathrm{M}$ single grating monochromator and detected by a nitrogen cooled photomultiplier coupled to a photon counting system.

The PL spectra of the samples A and B measured at $4.2 \mathrm{~K}$ are shown in Fig. 1. The low energy region of the spectra is dominated by sharp emission lines which originate from the bulk GaN layer. The lines observed in sample B are 


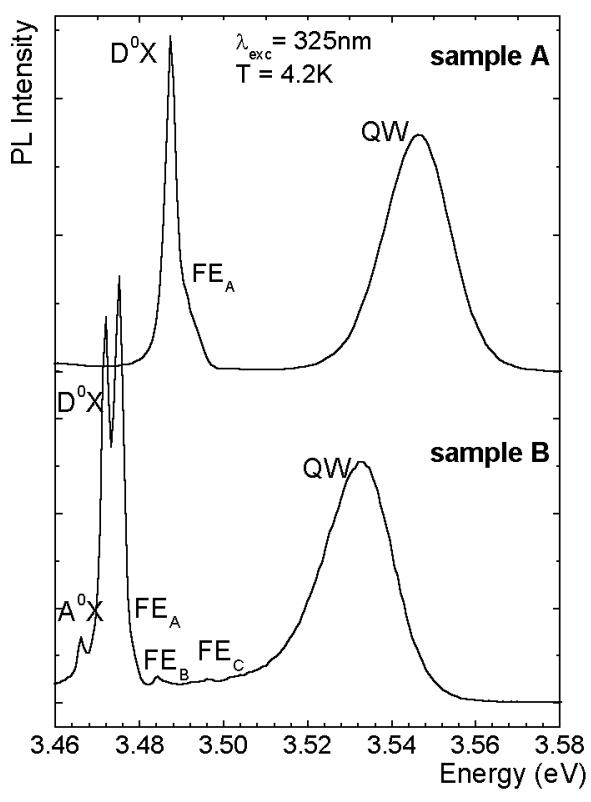

Fig. 1. PL spectra of the sample A and B at $4.2 \mathrm{~K}$.

better resolved, making possible a distinction between the emission due to recombination of $3 \mathrm{D}$ excitons bound to neutral donors (line $\mathrm{D}^{0} \mathrm{X}$ at $3.472 \mathrm{eV}$ ) and to neutral acceptors (line $\mathrm{A}^{0} \mathrm{X}$ at $3.466 \mathrm{eV}$ ). Moreover, in this sample the luminescence related to free excitons ( $\mathrm{FE}$ ) associated with the $\mathrm{A}, \mathrm{B}$, and $\mathrm{C}$ valence bands is observed. In contrast, the sample A exhibits only the donor-bound-exciton line (line $\mathrm{D}^{0} \mathrm{X}$ at $3.484 \mathrm{eV}$ ) shifted towards higher energy due to strain present in this heteroepitaxial structure. A small feature due to the free A exciton is also observed. Emissions related to the recombination of excitons in the QW appear at $3.546 \mathrm{eV}$ and $3.533 \mathrm{eV}$ in samples $\mathrm{A}$ and $\mathrm{B}$, respectively. The discrepancy of the QW recombination energies observed in both samples is caused by the difference in well width, strain, and build-in electric field. The full width at half maximum (FWHM) of both QW emissions is about $17 \mathrm{meV}$. This value is similar to the results obtained for analogous GaN/AlGaN QWs grown by MBE, exhibiting FWHM of 20-25 meV [4,5]. For the investigated structures, one monolayer step-like QW width fluctuations would result in an emission splitting of about $15 \mathrm{meV}$ [6]. Such behavior is not observed in the luminescence of both samples. Thus, we believe that in the investigated structures we are dealing with small size and high density interface roughness and/or compositional fluctuations in the barrier.

To investigate the carrier localization at the GaN/AlGaN QW interface we studied the temperature dependence of the QW emission. The PL spectra of samples A and B were measured at different temperatures, see Fig. 2. For both samples an anomalous temperature shift of the QW emission energy position was observed. 

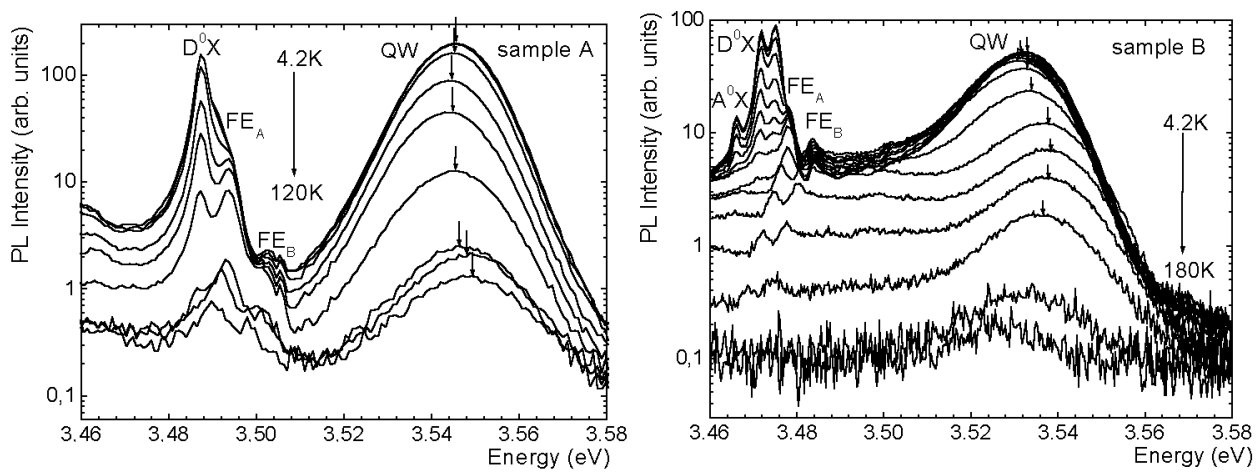

Fig. 2. PL spectra measured at different temperatures for the sample A (left part) and $\mathrm{B}$ (right part).
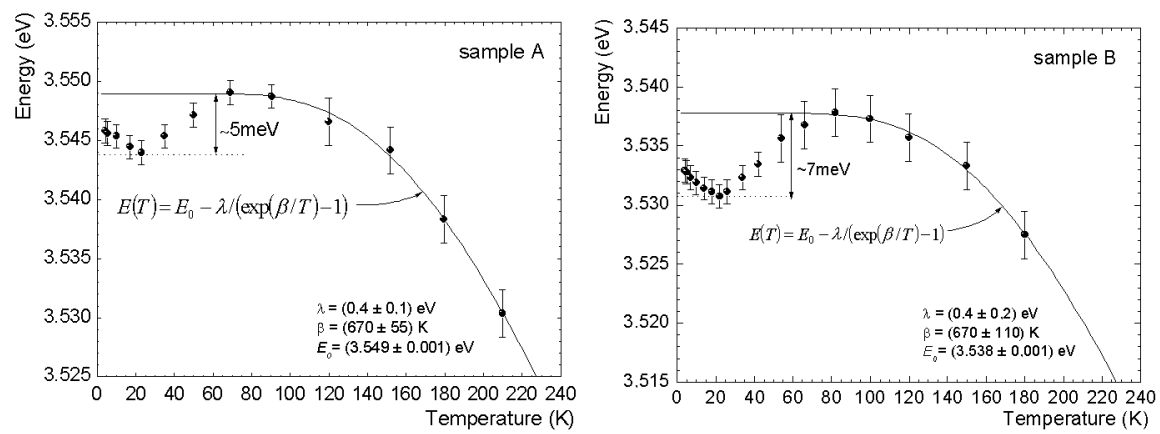

Fig. 3. Temperature dependence of the QW excitonic emission energy for the sample A (left part) and B (right part). The solid curves correspond to the best fit of Eq. (1).

The energy position corresponding to the emission maximum is plotted in Fig. 3. The energy of the QW emission decreases in the range of temperatures between $4.2 \mathrm{~K}$ and $20 \mathrm{~K}$ and then increases, reaching a maximum around $90 \mathrm{~K}$. Above this temperature the emission energy decreases again. The solid curves in Fig. 3 correspond to the best fit obtained at elevated temperatures using the formula [7]:

$$
E(T)=E_{0}-\lambda /[\exp (\beta / T)-1],
$$

where $\lambda, \beta$, and $E_{0}$ (the transition energy in the $0 \mathrm{~K}$ limit) are material dependent constants and here are fitting parameters. The curves match the data very well in the high temperature $(T>90 \mathrm{~K})$ regime. Thus, one can conclude that starting from a certain characteristic temperature the energy shift of the QW emission follows the changes of the QW material band gap, i.e. that of GaN. The values of the fitted parameters are $\lambda_{\text {sample } \mathrm{A}}=(0.4 \pm 0.1) \mathrm{eV}, \beta_{\text {sample } \mathrm{A}}=(670 \pm 55) \mathrm{K}$ and $\lambda_{\text {sample } \mathrm{B}}=(0.4 \pm 0.2) \mathrm{eV}, \beta_{\text {sample } \mathrm{B}}=(670 \pm 110) \mathrm{K}$ and are different than those reported for bulk GaN [7]: $\lambda=(0.121 \pm 0.005) \mathrm{eV}, \beta=(316 \pm 8) \mathrm{K}$. Such a difference can result from the strain present in a $\mathrm{QW}$ structure. 

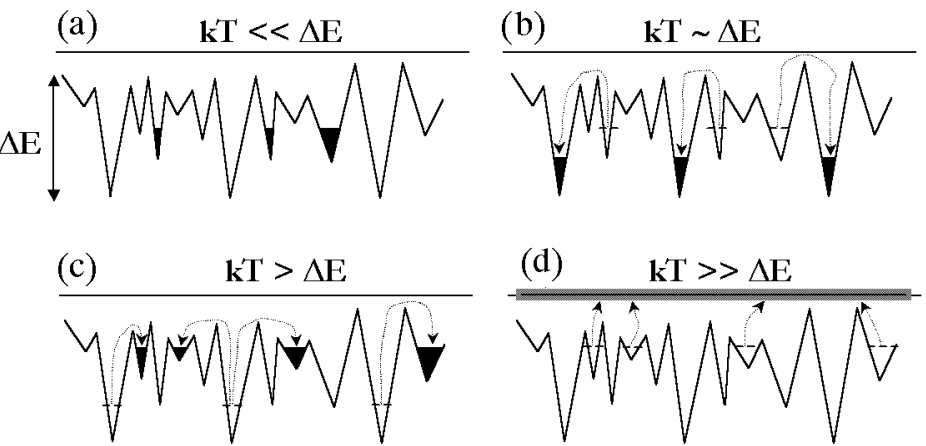

Fig. 4. Schematic diagrams illustrating occupation of the exciton traps at different temperatures (see text).

The behavior observed in the low temperature regime $(T<90 \mathrm{~K})$ can be explained in terms of the localization of the excitons in the QW. At low temperature $(T \approx 4.2 \mathrm{~K})$, the excitons are trapped by random potential fluctuations. These localized excitons mainly contribute to the luminescence since they do not have enough thermal energy to be activated (Fig. 4a). A slight increase in the temperature (up to $20 \mathrm{~K}$ ) allows the excitons to migrate. Thus, they can find local minima of the fluctuating potential (Fig. 4b), which leads to a decrease in the emission energy. Further increase in the temperature permits the release of the excitons from these traps (Fig. 4c). This results in an increase in the emission energy between $20 \mathrm{~K}$ and $90 \mathrm{~K}$. Above this temperature, the excitons are no longer localized (Fig. 4d) and the emission from the QW follows the temperature dependence of the $\mathrm{GaN}$ band gap.

The difference between the experimentally determined PL energies and those obtained from the fit of Eq. (1) allows to estimate the exciton localization energy. It is found in this way that the deepest traps should be up to $5-7 \mathrm{meV}$ below the free exciton level for both samples.

The obtained values of the characteristic temperature and localization energy seem to be independent of the growth method. Since the samples were deposited either on bulk GaN or on sapphire, it can be speculated that the localization of excitons cannot be attributed to structural defects. This suggests that the observed localization effects originate from the GaN/AlGaN interface itself. The observed disorder may be due to the material composition fluctuation and/or small size well width fluctuations. Also, the residual strain that induces an inhomogeneous electric field distribution in the structure can influence the localization of excitons. It is believed that the localization of holes plays a major role, since the spatial distribution of the electron wave function is too broad to penetrate small fluctuations of the potential [6]. The electron experiences an average potential originating from the interface disorder while the hole can be easy localized in potential traps due to the small extent of its wave function. 


\section{Conclusions}

In summary, we have studied the localization of free excitons in the $\mathrm{GaN} / \mathrm{AlGaN}$ QWs. In the low temperature regime the carriers are confined in traps up to 5-7 meV deep. By increasing the temperature above $90 \mathrm{~K}$, when $k T$ is in the range of the localization energy, the excitons become delocalized and the luminescence energy depends on temperature similarly to the behavior in bulk GaN. The observed S-shaped dependence is most probably caused by roughness and disorder of the GaN/AlGaN interface.

\section{Acknowledgments}

This work was partially supported by the State Committee for Scientific Research grant No. 2P03B 01122 and NATO Science Program.

\section{References}

[1] H.C. Ko, D.-C. Park, Y. Kawakami, S. Fujita, S. Fujita, Y.-S. Kim, Appl. Phys. Lett. 73, 1388 (1998).

[2] P. Tomasini, K. Arai, F. Lu, Z.Q. Zhu, T. Sekiguchi, T. Yao, M.Y. Shen, T. Goto, J. Appl. Phys. 83, 6028 (1998).

[3] Y.H. Cho, G.H. Gainer, A.J. Fisher, J.J. Song, S. Keller, U.K. Mishra, S.P. Den-Baars, Appl. Phys. Lett. 73, 1370 (1998).

[4] M. Leroux, N. Grandjean, M. Laügt, J. Massies, B. Gil, P. Lefebvre, P. Bigenwald, Phys. Rev. B 58, R13371 (1998).

[5] H. Haratizadeh, P.P. Paskov, G. Pozina, P.O. Holtz, B. Monemar, S. Kamiyama, M. Iwana, H. Amano, I. Kasaki, Appl. Phys. Lett. 80, 1373 (2002).

[6] M. Gallart, P. Lefebvre, A. Morel, T. Taliercio, B. Gil, J. Allegre, H. Mathieu, B. Damilano, N. Grandjean, J. Massies, Phys. Status Solidi A 183, 61 (2001).

[7] K.P. Korona, A. Wysmolek, K. Pakula, R. Stepniewski, J.M. Baranowski, I. Grzegory, B. Lucznik, M. Wroblewski, S. Porowski, Appl. Phys. Lett. 69, 788 (1995). 